\title{
Reprint
}

\section{The Effect of Finite Population on IEEE802.11 Wireless LANs Throughput/Delay Performance}

\author{
E. Ziouva and T. Antonakopoulos
}

The 2002 Mediterranean Electrotechnical Conference MELECON 2002

Copyright Notice: This material is presented to ensure timely dissemination of scholarly and technical work. Copyright and all rights therein are retained by authors or by other copyright holders. All persons copying this information are expected to adhere to the terms and constraints invoked by each author's copyright. In most cases, these works may not be reposted or mass reproduced without the explicit permission of the copyright holder. 


\title{
The Effect of Finite Population on IEEE802.11 Wireless LANs Throughput/Delay Performance
}

\author{
Eustathia Ziouva ${ }^{1}$ and Theodore Antonakopoulos ${ }^{2}$ \\ ${ }^{1}$ Computers Technology Institute, Riga Feraiou 61, 26221 Patras, Greece \\ ${ }^{2}$ Department of Electrical Engineering and Computers Technology,University of Patras, \\ 26500 Rio - Patras, Greece \\ Tel: +30-61-997 346, Fax: +30-61-997 342, e-mail: antonako@ee.upatras.gr
}

\begin{abstract}
The theoretical performance of CSMA/CA, as it is used in IEEE802.11 wireless LANs, is investigated in this paper. We adopt and modify a previously presented analytical approach for CSMA/CA protocols in wireless LANs with finite number of stations and find closed-form equations for throughput and delay. The presented numerical results highlight the characteristics of both CSMA/CA methods and define how their performance depends on the number of stations and on traffic conditions.
\end{abstract}

Keywords: IEEE802.11 WLANs, Carrier sense multiple access with collision avoidance, Distributed coordination function.

\section{INTRODUCTION}

The stations of a wireless local area network (WLAN) share the medium according to the IEEE802.11 standard [1]. The MAC protocol is based on the distributed coordination function (DCF) for asynchronous data transmission and on the centralized point coordination function (PCF) for time-bounded data transmissions. DCF is based on the CSMA/CA access method and a random backoff mechanism following each busy medium condition. Since the CSMA/CA can not rely on the stations capability to detect collisions by hearing their own transmission, there are two methods to determine the successful reception of a frame: the Basic CSMA/CA and the CSMA/CA with RTS/CTS exchange (RTS/CTS CSMA/CA).

Concerning the performance analysis of the CSMA/CA protocols, Zahedi [2] provides an approximate model to compute the throughput of an access point, while Chhaya [3] calculates the CSMA/CA throughput using a space dependent model. Bianchi [4] computes the saturation throughput of CSMA/CA protocol in the assumption of an ideal channel. Kim [5] considers the CSMA/CA as a hybrid protocol of slotted 1persistent CSMA and $p$-persistent CSMA and focuses on the performance of Access Points (APs) in infrastructure networks in Rayleigh and shadow fading channels. In [6] Bianchi revises and extends [4], providing a model that accounts for all the exponential backoff mechanism details. The condition under which the exponential backoff mechanism is activated is considered in the modified Bianchi's model presented in [7]. Moreover, [7] computes the saturation delay of CSMA/CA protocol. Finally, Cali [8] suggests a backoff algorithm for $p$-persistent protocols where the backoff interval is sampled from a geometric distribution with parameter $p$.

In most studies for CSMA protocols an infinite number of stations was considered for forming the total channel traffic as a Poisson process. This approach is unsuitable for a LAN with a relatively small number of stations. For a finite number of stations another approach is suggested by [9] in which a station is assumed to have idle periods (no packets) that are independent and geometrically distributed (in slotted CSMA protocols) and so the channel has the memoryless property. A different assumption is adopted in [4], [6], [7] and [8], where the finite number of stations operates in asymptotic conditions: a packet is always ready for transmission. In this work, we adopt the combination of slotted 1persistent and $p$-persistent protocols and we use the analytical approaches and assumptions of [5] and [9] in order to evaluate the performance of IEEE802.11 WLANs. In order to present a better insight to the performance of the different protocol modes, we do not consider the capture effect phenomenon, which results in a different closed-form equation for the time spent in successful transmissions. Our work assumes that the network consists of a small number of stations, no errors occur in the channel, no hidden terminal conditions are met and all data frames are of constant length. As in [5], we also use the renewal theory for the delay analysis, but the mean residual lifetime of a random variable is calculated differently.

Section 2 gives the throughput analysis of the CSMA/CA protocol and Section 3 continues with the delay analysis. Finally, Section 4 presents various numerical results and discusses how the protocol's performance is affected by its parameters. 


\section{Throughrut ANALYSIS}

If $\alpha$ is the backoff slot time per data frame transmission time, the time is considered slotted with slot size equal to $\alpha$, and the frame transmission time is considered as the unit time and all other time intervals are normalized to this time unit. In dealing with the case of a finite population (e.g. $M$ stations), we assume that the system state alternates between idle periods $(I)$ in which no station has frames to transmit and busy periods $(B)$ in which at least one station transmits a frame. The idle periods are assumed to be independent and geometrically distributed. Let $U$ be the time spent in useful transmission during a regeneration cycle. If $\bar{X}$ (or $E[X]$ ) denotes the expectation of a random variable $X$, then the system throughput $S$ is defined as [9]:

$$
S=\frac{\bar{U}}{\bar{B}+\bar{I}}
$$

Although the IEEE 802.11 MAC protocol uses either the Basic CSMA/CA or the RTS/CTS CSMA/CA, we consider also another reference method. In the so-called No-ACK CSMA/CA, the transmitter sends its data frame and does not wait for acknowledgement. The channel model of the CSMA/CA transmission methods is presented in Fig. 1 of ref. [5]. When a station transmits after an idle period $I$ (the backoff mechanism is not active yet), the CSMA/CA protocol is an adapted slotted 1persistent CSMA protocol, while for the rest of the busy period $B$ is an adapted slotted $p$-persistent protocol, where each ready station transmits with probability $p$. In [8] it is shown that the $p$-persistent IEEE802.11 protocol provides accurate estimates on the Basic CSMA/CA behavior if $p=1 /(E[W]+1)$, where $E[W]$ is the average backoff time. Additionally, we consider that each station generates a frame during each slot with probability $g$, which includes new arrivals and rescheduled frames. So, if $G$ is the offered load of all stations, then $g=\alpha G / M$ [9].

We use the methodology of [5] and [9] in order to derive the basic equations. The busy period is divided into several sub-busy periods. Each $j$ subbusy period is denoted by $B^{(j)}$ and is composed of the time interval DIFS, the transmission delay, $D^{(j)}$, which is due to the backoff procedure and the transmission time, $T^{(j)}$, which also includes the propagation delay $\tau$. The first sub-busy period $B^{(I)}$ consists of the transmission delay $D^{(l)}$, which is a DIFS delay and the transmission time $T^{(l)}$. For the No-ACK CSMA/CA the transmission time $T^{(j)}$ is equal to $1+\tau$ in all cases, even if the transmitted frame collides. A busy period continues if there is at least one station with a pending frame during the last transmission period or during the last DIFS time interval. We denote by $T P$ the sum of the last transmission period and the last DIFS, thus $T P=1+$ $\tau+f$. The idle period $I$ is geometrically distributed, therefore $\bar{I}=\alpha /\left[1-(1-g)^{M}\right]$.

The expectation of the delay introduced in each $j$ th sub-busy period $(j=2,3, \ldots)$ is given by:

$$
\begin{aligned}
& \overline{D^{(j)}}=\frac{\alpha}{1-(1-g)^{(T P / \alpha) M}}\left(\sum _ { k = 1 } ^ { \infty } \left\{(1-p)^{k}\right.\right. \\
& \left.-(1-g)^{T P / \alpha}\left[(1-p)^{k}-(1-g)^{k}\right]\right\}^{M} \\
& \left.-(1-g)^{(T P / \alpha) M} \sum_{k=1}^{\infty}(1-g)^{k M}\right)
\end{aligned}
$$

The sum of the expectation of the busy and the idle period is given by:

$$
\begin{aligned}
& \bar{B}+\bar{I}=f\left[1-(1-g)^{M}\right]+1+\tau \\
& +\frac{1}{(1-g)^{(T P / \alpha) M}}\left\{(f+1+\tau)\left[1-(1-g)^{(T P / \alpha) M}\right]\right. \\
& +\alpha \sum_{k=1}^{\infty}\left\{(1-p)^{k}-(1-g)^{(T P / \alpha)}\left[(1-p)^{k}-(1-g)^{k}\right]\right\}^{M} \\
& \left.-\alpha(1-g)^{(T P / \alpha) M} \sum_{k=1}^{\infty}(1-g)^{k M}\right\}+\frac{\alpha}{1-(1-g)^{M}}
\end{aligned}
$$

Since no capture effect is used, we have to calculate the expected value of the useful transmission period that is different from the one found in [5]. The $\overline{U^{(1)}}$ of the first sub-busy period is calculated considering that the transmission is successful during the first sub-busy period, if there is only one frame arrival in the last slot of the idle period. So

$\overline{U^{(1)}}=\frac{1}{1-(1-g)^{M}} M g(1-g)^{M-1}$

The expected value of the useful transmission period $U^{(j)}$, given that $N_{0}^{(j)}=n$ and $D^{(j)} \geq k \alpha$, is derived by considering that a transmission is successful when only one station transmits and no collision happens. If $k=0$ (there is at least a station with its backoff counter at zero) the transmission is successful when only one among $n$ stations with pending frames transmits. If $k>0$, the transmission is successful either when only one among $n$ stations with pending frames transmits or only one among $(M-n)$ empty stations has a new arrival, thus:

$$
\begin{aligned}
& E\left[U^{(j)} \mid D^{(j)} \geq k \alpha, N_{0}^{(j)}=n\right]= \\
& \begin{cases}n p(1-p)^{n-1} & k=0 \\
n p(1-p)^{n-1}+(M-n) g(1-g)^{M-n-1} & \\
-n(M-n) p g(1-p)^{n-1}(1-g)^{M-n-1} & k>0\end{cases}
\end{aligned}
$$


Using the theorem of total probability and summing the useful transmission periods for all sub-busy periods, we obtain:

$$
\begin{aligned}
& \bar{U}=\frac{1}{1-(1-g)^{M}} M g(1-g)^{M-1} \\
& +\left[\frac{1}{(1-g)^{(T P / \alpha) M}}-1\right] \sum_{n=1}^{M}\left\{n p(1-p)^{n-1}\right. \\
& +\left[n p(1-p)^{n-1}+(M-n) g(1-g)^{M-n-1}-n(M-n)\right. \\
& \left.\left.\cdot p g(1-p)^{n-1}(1-g)^{M-n-1}\right] \frac{(1-p)^{n}(1-g)^{M-n}}{1-(1-p)^{n}(1-g)^{M-n}}\right\} \\
& \left\{\frac{\left(\begin{array}{c}
M \\
n
\end{array}\right)\left[1-(1-g)^{T P / \alpha}\right]^{n}(1-g)^{(T P / \alpha)(M-n)}}{1-(1-g)^{(T P / \alpha) M}}\right\}
\end{aligned}
$$

Substituting (2.3) and (2.6) into (2.1), we get the channel throughput of the No-ACK CSMA/CA. The calculation of the channel throughput of the Basic and RTS/CTS CSMA/CA protocols is also based on the previous analysis. These access procedures differ from the No-ACK CSMA/CA in the time lengths of successful and non-successful transmissions. We define $T P_{S}$ as the sum of successful transmission time plus DIFS time and $T P_{F}$ as the sum of nonsuccessful transmission time plus DIFS time. We assume that the $j$ th transmission of the busy period is $X$ slots, so the length of the $(j+1)$ th transmission depends on whether the $j$ th transmission was successful or not. The length of the remaining busy period is a function of $X$. Let $B(X)$ be the mean duration of the busy period following the frame accumulation time of $X$ slots and $U(X)$ be the mean useful transmission time during the same busy period. We calculate $B(X)$ and $U(X)$ by using the following recursive consideration:

$$
\begin{aligned}
& B(X)=d(X) \\
& +\left\{T P_{S}+\left[1-(1-g)^{\left(T P_{S} / \alpha\right)}\right] B\left(T P_{S} / \alpha\right)\right\} u(X) \\
& +\left\{T P_{F}+\left[1-(1-g)^{\left(T P_{F} / \alpha\right)}\right] B\left(T P_{F} / \alpha\right)\right\}[1-u(X)] \\
& U(X)=\left\{1+\left[1-(1-g)^{\left(T P_{S} / \alpha\right)}\right] U\left(T P_{S} / \alpha\right)\right\} u(X) \\
& +\left\{\left[1-(1-g)^{\left(T P_{F} / \alpha\right)}\right] U\left(T P_{F} / a\right)\right\}[1-u(X)]
\end{aligned}
$$

where $d(X)$ and $u(X)$ are obtained by (2.2) and (2.5) respectively.

For $j \geq 1$ the busy and the useful transmission periods depend on the number of frame arrivals during the last slot of the idle period, thus $\bar{B}=B(1)$ and $\bar{U}=U(1)$ and from (2.1) it follows that

$$
S=\frac{U(1)}{B(1)+\frac{\alpha}{\left[1-(1-g)^{M}\right]}}
$$

For finding the throughput $S$, we have to calculate $B\left(T P_{S} / \alpha\right), B\left(T P_{F} / \alpha\right), U\left(T P_{S} / \alpha\right)$ and $U\left(T P_{F} / \alpha\right)$. Using (2.7) for $X=T P_{S} / \alpha$ and for $X=T P_{F} / \alpha$, we obtain two equations of two unknowns, $B\left(T P_{S} / \alpha\right)$ and $B\left(T P_{F} / \alpha\right)$. Solving these equations and substituting $d(1)$ and $u(1)$ we can calculate $B(1)$. Using (2.8) and the above described procedure, we can calculate $U(1)$. The duration of successful and non-successful transmission plus the DIFS interval of the Basic CSMA/CA and the RTS/CTS CSMA/CA are respectively given by:

$$
\begin{array}{ll}
\text { Basic: } \quad & T P_{S}=1+\beta+\delta+2 \tau+f \\
& T P_{F}=1+\tau+f \\
\text { RTS/CTS: } & T P_{S}=1+\gamma+\theta+\delta+3 \beta+4 \tau+f \\
& T P_{F}=\gamma+\tau+f
\end{array}
$$

where $\beta$ is the normalized length of SIFS, $\delta$ the normalized length of an ACK frame, $\gamma$ is the normalized length of an RTS frame and $\theta$ the normalized length of a CTS frame.

\section{DELAY ANALYSIS}

As frame delay $L$ is defined the time elapsed between the generation of a frame and its successful reception and is measured in frame transmission times. The backoff algorithm and the number of retransmissions are the main parameters that affect the frame delay. If a frame is received successfully the first time it is transmitted, then the delay depends on the frame transmission time $T$ and the medium access time $R . R$ is defined as the time elapsed from the moment a station starts sensing the medium to the moment it accesses the medium (due to the backoff procedure). For calculating the average frame delay for the Basic and RTS/CTS CSMA/CA, we define as $T_{S}$ the duration of a successful transmission period $\left(T P_{S}\right.$ duration minus DIFS) and as $T_{F}$ the duration of a non-successful transmission period ( $T P_{F}$ duration minus DIFS). The probability of a successful transmission is $P_{\text {Succ }}=S / G$, thus the probability of a non-successful transmission is $P_{\text {Fail }}=1-P_{\text {Succ }}$. Then the mean value of the medium access time $R$ is calculated [7] by:

$\bar{R}=$

$$
\begin{aligned}
& \frac{\bar{I}}{\bar{B}+\bar{I}} f+\frac{\bar{D}}{\bar{B}+\bar{I}} f+\frac{\bar{B}-\bar{D}}{\bar{B}+\bar{I}}\left\{P_{\text {Succ }}\left[\overline{\frac{\left(T_{S}+f+\Delta\left(T P_{S}\right)\right)^{2}}{2 \overline{\left(T_{S}+f+\Delta\left(T P_{S}\right)\right)}}}\right]\right. \\
& \left.+P_{\text {Fail }}\left[\overline{\frac{\left(T_{F}+f+\Delta\left(T P_{F}\right)\right)^{2}}{2 \overline{\left(T_{F}+f+\Delta\left(T P_{F}\right)\right)}}}\right]\right\}
\end{aligned}
$$


where $\overline{(T+f+\Delta(X))^{2}} / 2 \overline{(T+f+\Delta(X))}$ (different from the value calculated in [5]) is the mean residual life of the time interval $T+f+\Delta(X)$ between two successive accesses to the medium. Since $T$ and $f$ are constants:

$\overline{(T+f+\Delta(X))}=T+f+\overline{\Delta(X)}$

$\overline{(T+f+\Delta(X))^{2}}=$

$(T+f)^{2}+2(T+f) \overline{\Delta(X)}+\overline{(\Delta(X))^{2}}$

$\overline{\Delta(X)}$ is calculated by (2.2) for $X=T P_{S}$ and $X=T P_{F}$, while $\overline{(\Delta(X))^{2}}$ is obtained by the following equation

$$
\begin{aligned}
& \overline{\left(\Delta(X)^{(j)}\right)^{2}}=\frac{a^{2}}{1-(1-g)^{(T P / a) M}} \cdot\left(\sum_{k=1}^{\infty}(2 k-1)\right. \\
& \left\{(1-p)^{k}-(1-g)^{T P / a}\left[(1-p)^{k}-(1-g)^{k}\right]\right\}^{M} \\
& \left.-(1-g)^{(T P / a) M} \sum_{k=1}^{\infty}(2 k-1)(1-g)^{k M}\right)
\end{aligned}
$$

Finally, $\bar{D}$ is equal to $D(1)$ which can be obtained by

$$
\begin{aligned}
& D(1)=f+\left\{d\left(T P_{S}\right)+\left[1-(1-g)^{\left(T P_{S} / \alpha\right)}\right] D\left(T P_{S}\right)\right\} u(1) \\
& +\left\{d\left(T P_{F}\right)+\left[1-(1-g)^{\left(T P_{F} / \alpha\right)}\right] D\left(T P_{F}\right)\right\}[1-u(1)]
\end{aligned}
$$

The mean number of collisions is calculated by using throughput $S$ and the total traffic $G . G / S$ is the mean number of trials for transmitting a frame successfully, so the mean number of collisions is equal to $(G / S-1)$. Therefore the average frame delay for the Basic and RTS/CTS method is obtained by

$$
\bar{L}=\left(\frac{G}{S}-1\right)\left(T_{F}+\bar{Y}+\bar{R}\right)+\left(T_{S}+\bar{R}\right)
$$

where $Y$ is a random variable that represents the time a station waits until a frame collision has been detected.

\section{NUMERICAL RESULTS}

In this section, we present numerical results for channel throughput and frame delay of both types of CSMA/CA and we compare their performance. The parameters for our numerical calculations are: Frame payload $=1023$ bytes, MAC header $=34$ bytes (including the FCS field), PHY header $=16$ bytes, $\mathrm{ACK}=14$ bytes + PHY header, RTS $=20$ bytes + PHY header, CTS $=14$ bytes + PHY header, SIFS $=$
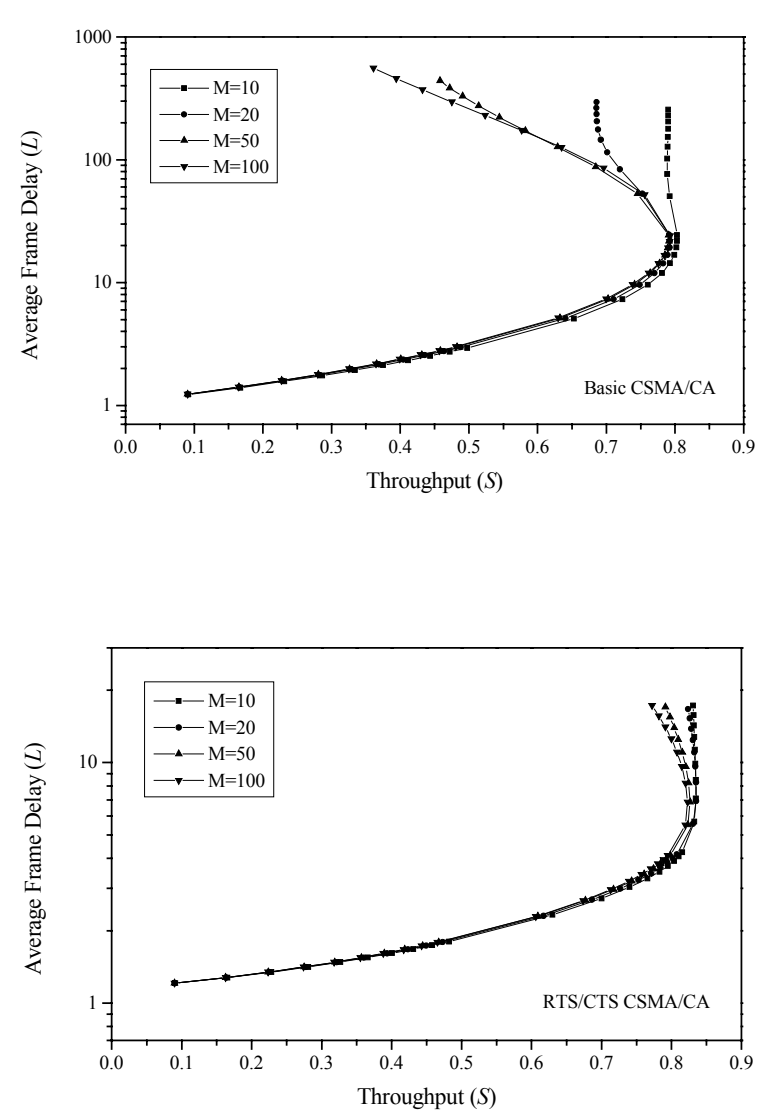

Figure 4.1. Delay-throughput tradeoff $(p=0.03)$

10 us, DIFS $=50$ us, propagation delay $=1$ us, slot time $=20$ us and channel bit rate $=5.5 \mathrm{Mbps}$.

Figure 4.1 illustrates the delay versus the throughput of the Basic and the RTSCTS CSMA/CA for different numbers of stations. Note that the performance of the Basic method deteriorates rapidly (low throughput and large delays) as the number of stations increases due to the increased collision probability. The RTS/CTS method provides higher throughput and much lower delay. As the number of stations increases, the performance of this method appears more robust. The enhanced performance of RTS/CTS method is due to the reduced duration of retransmissions.

In Figure 4.2 is depicted the dependence of the CSMA/CA methods on the traffic $G$ and the transmission probability $p$. The throughput of the Basic method decreases in high load conditions and when the transmission probability $p$ increases, since lower average backoff time is experienced and the collision probability increases. However, the RTS/CTS method provides significantly better throughput at high load conditions and is less dependable on the backoff procedure. 


\section{CONCLUSIONS}

In this paper, we analyzed and compared the Basic and the RTS/CTS modes of the CSMA/CA protocol. The Basic method has low throughput and large delays at high loads and depends strongly on the number of stations and the transmission probability. On the other hand, the RTS/CTS method provides higher throughput and lower delays when the system is highly loaded and is more robust to fluctuations in the number of stations and the transmission probability.

\section{References}

[1] P802.11, Draft Standard for wireless LAN medium access control (MAC) and physical layer (PHY) specification, IEEE, May 1997.

[2] Zahedi, A. and Pahlavan, K.,"Throughput of a wireless LAN access point in presence of natural hidden terminals and capture effects," In Proc. IEEE PIMRC, Taipei, Taiwan, 1996, pp. 397-401.

[3] Chhaya, H. S. and Gupta, S., "Performance modeling of asynchronous data transfers of IEEE 802.11 MAC protocol," Wireless Networks, vol. 3, 1997, pp. 217-234.

[4] Bianchi, G., "IEEE 802.11 - Saturation Throughput Analysis," IEEE Commun. Lett., vol. 2, no. 12, December 1998, pp. 318-320.

[5] Kim, J. H. and Lee, J. K., "Capture Effects of Wireless CSMA/CA Protocols in Rayleigh and Shadow Fading channels," IEEE Trans. Veh. Technol., vol. 48, no. 4, July 1999, pp. 1277-1286.

[6] Bianchi, G., "Performance Analysis of the IEEE 802.11 Distributed Coordination Function," IEEE J. Select. Areas Commun., vol. 18, no. 3, March 2000, pp. 535-547.

[7] Ziouva, E. and Antonakopoulos, T., "CSMA/CA performance under high traffic conditions: Throughput and delay analysis," Computer Communications, vol. 25, March 2002, pp. 313-321.

[8] Cali, F., Conti, M. and Gregori, E., "Dynamic Tuning of the IEEE 802.11 Protocol to Achieve a Theoretical Throughput Limit," IEEE/ACM Trans. Networking, vol. 8, no. 6, December 2000, pp. 785-799.

[9] Takagi, H. and Kleinrock, L., "Throughput analysis for CSMA systems," IEEE Trans.Commun., vol. COM-33, no. 7, July 1985, pp. 627-638.
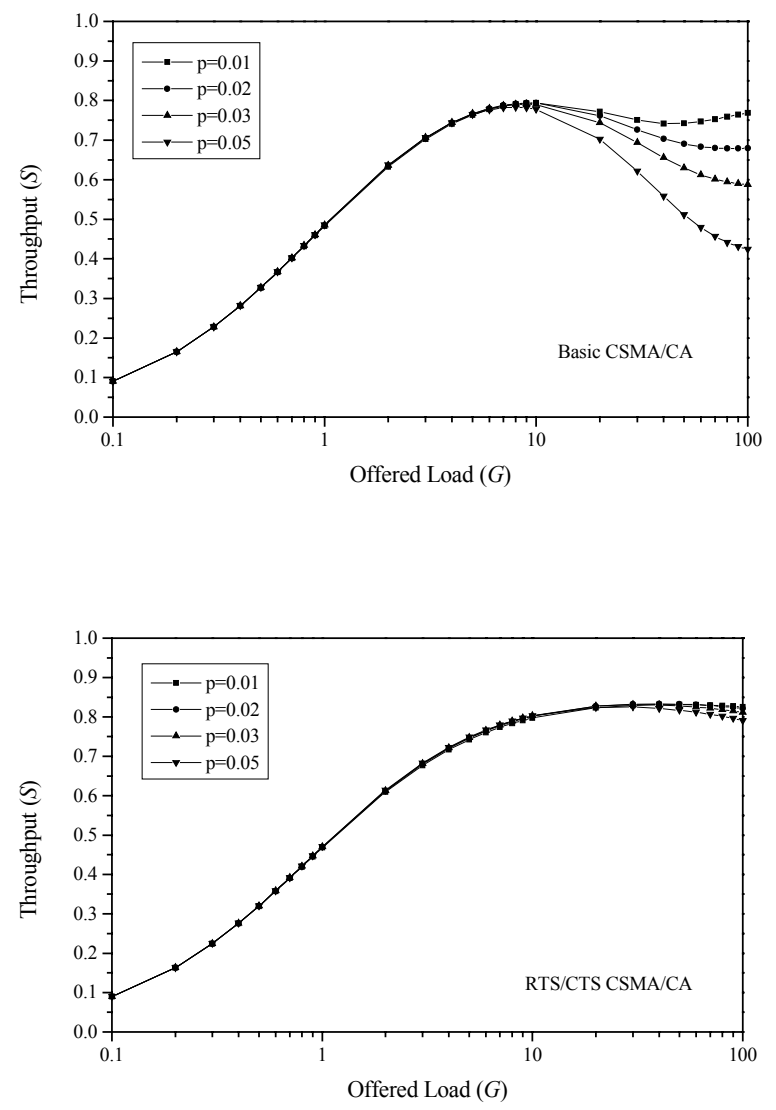

Figure 4.2. The Effect of backoff procedure $(M=50)$ 